\title{
Improving Dynamic Response Accuracy of Conventional Lumped-Mass Stick Model
}

\author{
Hwasung Roh ${ }^{\mathrm{a}, *}$, Hwichang Sun ${ }^{\mathrm{b}}$ \\ ${ }^{a}$ Associate Professor, Chonbuk National University, 567 Baekje-daero, Jeonju, 54896, Republic of Korea \\ ${ }^{\mathrm{b}}$ Graduate Research Assistant, Chonbuk National University, 567 Baekje-daero, Jeonju, 54896, Republic of Korea \\ *Corresponding Author: hwasung@jbnu.ac.kr
}

\begin{abstract}
The dynamic responses of tributary area based lumped-mass stick models may differ from the results of the actual model since their natural frequencies and eigenvectors are not identical to the actual model such as finite element (FE) model and real structures. This paper is to introduce an advanced technique to minimize the differences by employing the modal response $D(t)$. The parameter $D(t)$ depends on modal frequency and damping, but independent on the mode shapes (eigenvectors) of structures. The identical response $D(t)$ is obtained from the frequency adaptive LMS model which provides identical modal frequencies as the actual model. In order to show the performance of the advanced technique, a straight column is considered. From the time history response results, the technique improves the dynamic response accuracy of the conventional LMS model.
\end{abstract}

Keywords: lumped-mass stick models, dynamic response accuracy, modal frequency, eigenvectors

\section{Introduction}

Due to the simplicity and more modeling efficiency, the LMS models have been widely used in seismic response analysis of Nuclear Power Plants (NPPs) ${ }^{(1-2)}$. Also, the LMS models are used in other fields such as seismic analysis of bridges $^{(3-5)}$. Other researchers have employed the LMS model to run seismic analysis on tall buildings ${ }^{(6)}$, electric bushings ${ }^{(7)}$ and electricity transmission towers ${ }^{(8)}$. However, the accuracy of the LMS model has been brought to question. Radford et al. ${ }^{(9)}$ investigated seismic responses between the FE and LMS models and indicated that the
LMS model cannot accurately represent FE model for higher frequencies dominated structure. Mainly, the difference in seismic responses between the LMS model and the FE model occurs because of the difference in natural frequencies between the two models.

The natural frequencies and eigenvectors of the conventional LMS model are usually not the same as the FE model or actual structures, especially for higher modes. Due to this, two kinds of error occur as result of the LMS model, which are period and amplitude errors in the displacement and acceleration time history responses. The purpose of the study is to improve the response accuracy of the conventional LMS model by employing identical responses $D(t)$ as the actual structure.

\section{Advanced Technique of LMS Model}

\subsection{Conventional LMS Model}

In the conventional LMS model, each lumped mass is determined considering the geometric configuration of the actual model, which is called "tributary area consideration" ${ }^{(10)}$. For the multi-degrees of freedom system of the conventional LMS model, the equation of motion for the $n$th mode is expressed as

$$
\ddot{q}_{n}(t)+2 \xi_{n} \omega_{n} \dot{q}_{n}(t)+\omega_{n}^{2} q_{n}(t)=\Gamma_{n} \ddot{u}_{g}(t)
$$

where $q_{n}, \xi_{n}, \omega_{n}$, and $\Gamma_{n}$ are the generalized modal coordinate, modal damping ratio, modal natural frequency and modal participation factor, respectively. The parameter $\ddot{u}_{g}$ is ground acceleration. The modal participation factor $\left(\Gamma_{n}\right)$ is expressed as $\Gamma_{n}=\phi_{\text {mass }, n}^{T} \mathbf{M} \ell$ where $\mathbf{M}$ is the 
mass matrix, and $\ell$ is the influence vector. If the maximum normalized eigenvectors $\left(\phi_{n}\right)$ is used, the parameter $\Gamma_{n}$ is defined as $\Gamma_{n}=\tilde{m}_{n}^{-1} \phi_{n}^{T} \mathbf{M} \ell$, where $\tilde{m}_{n}$ is the modal mass (generalized mass) of the $n$th mode as result of the maximum normalized eigenvectors. As shown Eq. (1), the responses obtained from the conventional LMS model are different with the actual model because natural frequencies of the conventional model are not same as the actual model. Also, difference between the LMS and actual model could occur due to the difference in mass and eigenvector matrices which are involved in the modal participation factor.

\subsection{Updating Conventional LMS Model}

It is well known that the coordinate $q_{n}$ is defined by introducing the parameter $D_{n}(t)$ as shown in Eq. (2).

$$
q_{n}(t)=\Gamma_{n} D_{n}(t)
$$

Thus, the dynamic system equation, Eq. (1), is re-written in terms of the parameter $D_{n}(t)$ as follows.

$$
\ddot{D}_{n}(t)+2 \xi_{n} \omega_{n} \dot{D}_{n}(t)+\omega_{n}^{2} D_{n}(t)=\ddot{u}_{g}(t)
$$

Eq. (3) is the form of Single Degree of Freedom (SDF) system where the response $D_{n}(t)$ is dependent only on the modal natural frequency and damping ratio, not dependent on the mass distribution of the multi-degrees of freedom (MDF) system. Eq. (3) can be calculated by using Duhamel integration using the Newmark method or other numerical methods $^{(10)}$. For the MDF system, the generalized coordinate $\left(q_{n}\right)$ is expressed in matrix form as shown in Eq. (4). Therefore, the relative displacement is written as Eq. (5).

$$
\begin{gathered}
\mathbf{q}(t)=\boldsymbol{\Gamma} \mathbf{D}(t) \\
\mathbf{u}(t)=\boldsymbol{\Phi} \boldsymbol{\Gamma} \mathbf{D}(t)
\end{gathered}
$$

where $\boldsymbol{\Phi}$ is the maximum normalized eigenvector matrix, $\boldsymbol{\Gamma}$ is a diagonal square matrix of the modal participation factors and $\mathbf{D}(t)$ is a single column vector which consist of the parameters $D_{n}(t)$.

$$
\boldsymbol{\Gamma}=\left[\begin{array}{cccc}
\Gamma_{1} & & & \\
& \Gamma_{2} & & \\
& & \ddots & \\
& & & \Gamma_{n}
\end{array}\right] ; \mathbf{D}(t)=\left[\begin{array}{c}
D_{1}(t) \\
D_{2}(t) \\
\vdots \\
D_{n}(t)
\end{array}\right]
$$

If the response $\mathbf{D}(t)$ of the actual model is employed, the period or frequency error is reduced to zero. Instead of the use of Duhamel integration, alternative way to obtain the identical response $\mathbf{D}(t)$ is to use the frequency adaptive method which is developed by Roh et al. ${ }^{(11)}$ and Lee et al. ${ }^{(12)}$. Detailed things related to the frequency adaptive method can be found in the references ${ }^{(11,12)}$. Using the frequency adaptive method, the relative displacement at each node is presented as follows.

$$
\mathbf{u}_{f}(t)=\boldsymbol{\Phi}_{f} \boldsymbol{\Gamma}_{f} \mathbf{D}_{f}(t)
$$

Here, the parameter $\mathbf{D}_{f}(t)$ is the response $\mathbf{D}(t)$ obtained from the frequency adaptive method and calculated by using Eq. (8).

$$
\mathbf{D}_{f}(t)=\left(\boldsymbol{\Phi}_{f} \boldsymbol{\Gamma}_{f}\right)^{-1} \mathbf{u}_{f}(t)
$$

The response $\mathbf{D}_{f}(t)$ is exactly the same as the actual model. Also, the displacement response of the conventional LMS model is updated by inserting the identical response of the actual structure, $\mathbf{D}_{f}(t)$, into Eq. (5), which is presented as follows.

$$
\mathbf{u}_{u p}(t)=\boldsymbol{\Phi} \Gamma \mathbf{D}_{f}(t)=\boldsymbol{\Phi} \boldsymbol{\Gamma}\left(\boldsymbol{\Phi}_{f} \boldsymbol{\Gamma}_{f}\right)^{-1} \mathbf{u}_{f}(t)
$$

where $\mathbf{u}_{u p}(t)$ is the updated displacement response of the conventional LMS model. The updated total acceleration response of the conventional LMS model, $\ddot{\mathbf{u}}_{u p}^{t}(t)$, is expressed as follows.

$$
\ddot{\mathbf{u}}_{u p}^{t}(t)=\ddot{u}_{g}(t) \ell+\Phi \Gamma \ddot{\mathbf{D}}_{f}(t)
$$

The updated displacement and acceleration responses have a zero period error since the responses $\mathbf{D}_{f}(t)$ and $\ddot{\mathbf{D}}_{f}(t)$ are identical to those of the actual structure. 


\section{Case Study-Straight Column}

A uniform-straight column is selected in order to show the performance of the advanced or updating technique for the conventional LMS model responses. The concrete straight column shown in Fig. 1 is presented by FE model which is assumed as actual structure in this paper. Total amount of mass is $6118.2 \mathrm{~kg}$, total height is $10 \mathrm{~m}$, and a cross section area is $0.5 \mathrm{~m}$ by $0.5 \mathrm{~m}$. The elastic modulus and shear modulus are $25 \mathrm{GPa}$ and $12.5 \mathrm{GPa}$, respectively. The selection of modes is related to the number of masses and determined according to the modal participating mass ratio where normally $90 \%$ or above. In this example six lumped masses are selected from the eigenvalue analysis since the sixth mode covers up to $94 \%$ of the modal participating mass ratio. The conventional LMS model consisting six nodes is shown in Fig. 1. The lumped masses are calculated from the tributary areas basis.
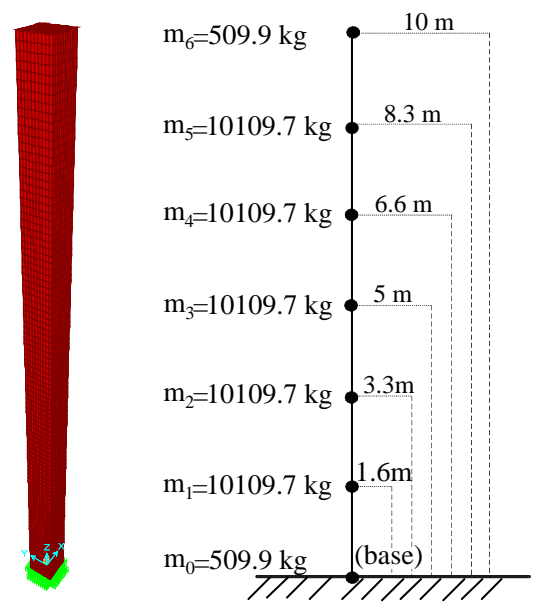

Fig. 1. Finite element model of straight column and conventional lumped-mass distribution.

From the eigenvalue analysis, the eigenvector and modal participation factor matrices are presented in Eq. (11) and Eq. (12), respectively. The error of the conventional LMS model in frequency especially for higher modes can be seen in Table 1. The frequency adaptive LMS model is built considering six lumped masses and six stick elements. Same stick elements of the conventional model are used. To obtain identical modal frequencies and corresponding response $\mathbf{D}(t)$, however, the amounts of lumped mass for each node are determined through the iterative method ${ }^{(11,12)}$. From the eigenvalue analysis, Table 1 compares the natural frequencies of the FE model with those of the conventional and frequency adaptive LMS models, showing that the error of the frequency adaptive LMS model is almost zero for all selected modes.

$$
\boldsymbol{\Phi}=\left[\begin{array}{cccccc}
0.045 & -0.236 & 0.586 & -0.994 & 1 & 0.797 \\
0.165 & -0.616 & 0.897 & -0.385 & -0.585 & -0.970 \\
0.338 & -0.760 & 0.123 & 1 & -0.200 & 1 \\
0.546 & -0.480 & -0.736 & 0.021 & 0.848 & -0.835 \\
0.770 & 0.171 & -0.408 & -0.901 & -0.790 & 0.489 \\
1 & 1 & 1 & 0.905 & 0.530 & -0.270
\end{array}\right]
$$

$$
\boldsymbol{\Gamma}=\left[\begin{array}{llllll}
1.5408 & & & & & \\
& -0.8018 & & & & \\
& & 0.4054 & & & \\
& & & -0.2402 & & \\
& & & & 0.1874 & \\
& & & & & 0.0975
\end{array}\right]
$$

Table 1. Natural frequencies of FE and LMS models

\begin{tabular}{c|c|c|c|c|c}
\hline \multirow{2}{*}{ Mode } & \multicolumn{3}{|c|}{ Natural frequency (Hz) } & \multicolumn{2}{c}{ Error (\%) } \\
\cline { 2 - 6 } & FE & Conventional & $\begin{array}{c}\text { Frequency } \\
\text { adaptive }\end{array}$ & Conventional & $\begin{array}{c}\text { Frequency } \\
\text { adaptive }\end{array}$ \\
\hline 1st & 2.58 & 2.55 & 2.58 & 1.21 & $4.4 \mathrm{E}-08$ \\
2nd & 16.00 & 15.38 & 16.00 & 3.86 & $3.8 \mathrm{E}-08$ \\
3rd & 44.12 & 41.46 & 44.12 & 6.03 & $9.6 \mathrm{E}-08$ \\
4th & 84.66 & 77.38 & 84.66 & 8.61 & $4.5 \mathrm{E}-08$ \\
5th & 136.42 & 118.35 & 136.42 & 13.25 & $5.3 \mathrm{E}-08$ \\
6th & 197.91 & 152.76 & 197.91 & 22.81 & $1.6 \mathrm{E}-08$ \\
\hline
\end{tabular}

For the time history analysis, a constant damping ratio of $2 \%$ and whitenoise excitation are considered. The duration of the whitenoise is 30 seconds and it covers $200 \mathrm{~Hz}$. The time history analysis is performed for the frequency adaptive LMS model and the lateral displacement responses for each node, which is $\mathbf{u}_{f}(t)$ and $\ddot{\mathbf{u}}_{f}(t)$, is obtained. Using Eq. (8), the responses $\mathbf{D}_{f}(t)$ is obtained and shown in Fig. 2.

The peak responses of the conventional model before and after updating for each node are compared with those of their corresponding locations in the FE model. In this study, dynamic responses of the conventional LMS model after updating are referred as updated LMS responses. Table 2 shows the peak displacement at each node of the FE model and the conventional and updated LMS responses. The peak displacement after updating shows better result than that the conventional model.

Fig. 3 shows the displacement time history responses of the conventional and updated Lumped-Mass Stick (LMS) models compared with the Finite Element (FE) model, capturing the responses from 2 to 8 seconds. 


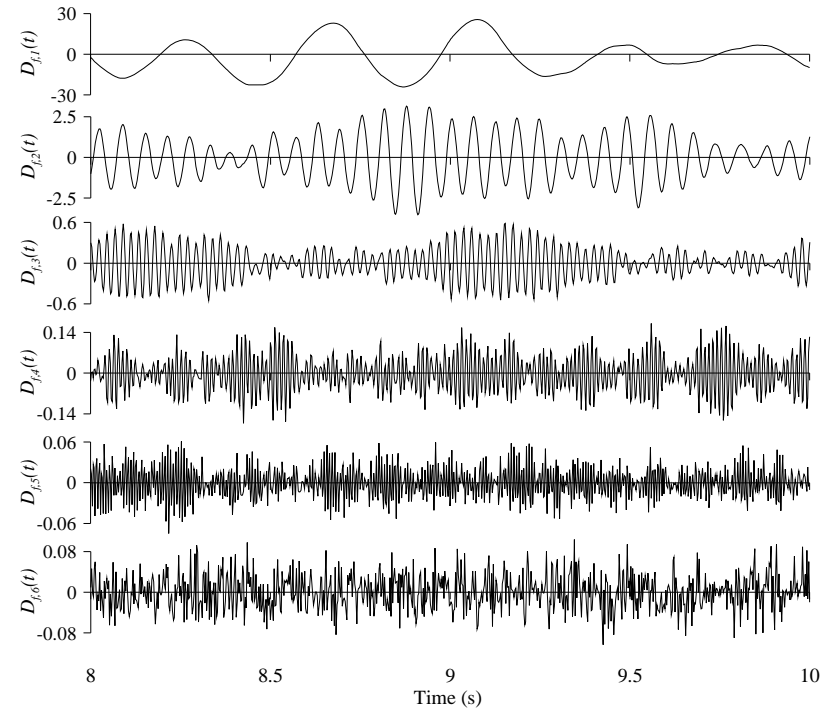

Fig. 2. Response $\mathbf{D}_{f}(t)$ from frequency adaptive LMS model (from 8 to 10 seconds as an example).

Table 2. Comparison of peak displacement response

\begin{tabular}{c|ccc|cc}
\hline & FE & Conventional & Updated & Conventional & Updated \\
\hline Node & \multicolumn{2}{|c|}{ Peak displacement (mm) } & \multicolumn{2}{c}{ Error (\%) } \\
\hline 1 & 3.310 & 3.430 & 3.109 & 3.63 & 6.09 \\
2 & 11.130 & 12.070 & 11.091 & 8.45 & 0.35 \\
3 & 22.720 & 23.970 & 22.281 & 5.50 & 1.93 \\
4 & 36.430 & 37.900 & 35.510 & 4.04 & 2.53 \\
5 & 50.620 & 52.850 & 50.057 & 4.41 & 1.11 \\
6 & 66.550 & 68.190 & 65.511 & 2.46 & 1.56 \\
\hline
\end{tabular}

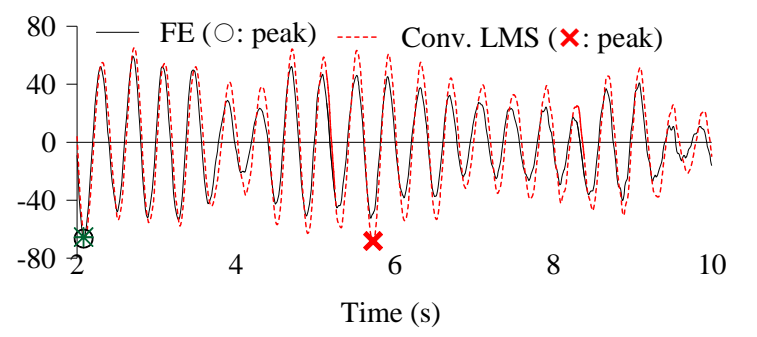

(a) FE versus Conventional LMS

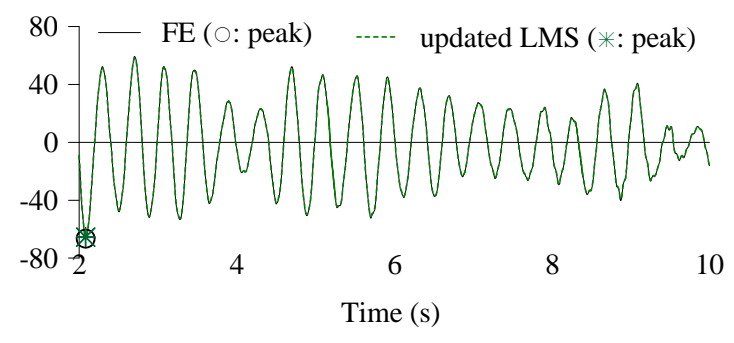

(b) FE versus updated LMS

Fig. 3. Comparison of displacement response at the top captured for 2 to 10 seconds
It is clearly shown that the phase error is reduced to zero for the updated LMS response where the peak displacement of the FE model and updated response occurs at the same time which is 2.1 seconds. For the peak acceleration response shown in Table 3, the accuracy of the updated response also provides better result compared to that of the conventional LMS response.

Table 3. Comparison of peak acceleration response

\begin{tabular}{c|ccc|cc}
\hline & FE & Conventional & Updated & Conventional & Updated \\
\hline Node & \multicolumn{2}{|c|}{ Peak absolute acceleration (g) } & \multicolumn{2}{|c}{ Error (\%) } \\
\hline 1 & 3.370 & 2.712 & 3.165 & 19.53 & 6.10 \\
2 & 3.199 & 3.336 & 3.166 & 4.27 & 1.04 \\
3 & 3.492 & 3.235 & 3.384 & 7.36 & 3.09 \\
4 & 3.103 & 3.198 & 2.907 & 3.08 & 6.30 \\
5 & 3.021 & 2.868 & 3.033 & 5.07 & 0.39 \\
6 & 6.791 & 4.948 & 5.439 & 27.14 & 19.91 \\
\hline
\end{tabular}

The procedures of the updating method are not much complex since all required steps are based on modal response and matrix combination which can be simply operated with MALAB or equivalent numerical platform.

\section{Conclusions}

The advanced technique is proposed to improve the dynamic response accuracy of the conventional LMS model. The technique is based on the responses $\mathbf{D}_{f}(t)$ and $\ddot{\mathbf{D}}_{f}(t)$ of the actual model. The frequency adaptive LMS model is adopted to obtain the identical responses $\mathbf{D}_{f}(t)$ and $\ddot{\mathbf{D}}_{f}(t)$ to the actual model. From the response investigation of the straight column, the peak dynamic responses of the conventional model before and after updating are compared with those of FE (actual) model at each node. The peak responses after updating are much closer to the FE model. Furthermore, the phase or period error of the time history results of the updated LMS response is almost zero since their modal frequencies match with the frequencies of the FE model.

\section{Acknowledgment}

This research was supported by Basic Science Research Program of National Research Foundation of Korea (NRF) fully funded by the Ministry of Education, Korea (Grant No: 2015R1D1A3A01020017). 


\section{References}

(1) TS Leonardo, SO Richard, T Sener and PR Diego : "Finite Element Modeling of the AP1000 Nuclear Island for Seismic Analysis at Generic Soil and Rock Sites”, Nuclear Engineering and Design, Vol. 237, No. 12-13, pp. 1474-1485, 2007.

(2) LM Anderson, A Hashemi and F Ostadan : "Soil-Structure Interaction Effects on Nuclear Structures Founded on Rock Sites," Proceeding of the 10th National Conference in Earthquake Engineering, Earthquake Engineering Research Institute, Anchorage, Alaska, 2014.

(3) JY Meng and EM Lui : "Refined Stick Model for Dynamic Analysis of Skew Highway Bridges,” Journal of Bridge Engineering, Vol. 7, No. 3, pp. 184-194, 2002.

(4) S Agrawal and AK Jain : "Seismic Analysis of a S-Curved Viaduct using Stick and Finite Element models," International Journal of Civil, Environmental, Structural, Construction and Architectural Engineering, Vol. 3, No. 2, pp. 34-44, 2009.

(5) E Amirihormozaki, G Pekcan and A Itandi : "Analytical Modeling of Horizontally Curved Steel Girder Highway Bridges for Seismic Analysis," Journal of Earthquake Engineering, Vol. 19, No. 2, pp. 220-248, 2015.

(6) L Huo, C Qu and $\mathrm{H} \mathrm{Li}$ : "TLCD Parametric Optimization for the Vibration Control of Building Structures Based on Linear Matrix Inequality,” Journal of Applied Mathematics, Vol. 2014, Article ID. 527530, pp. 10, 2014.

(7) H Roh, ND Ovileto and AM Reinhorn : "Experimental Test and modeling of Hollow-Core Composite Insulators," Nonlinear Dynamics, Vol. 69, No. 4, pp. 1651-1663, 2012.

(8) BO Chen, WH Guo, PY Li and WP Xie : "Dynamic Response and Vibration Control of the Transmission Tower-Line System: A State-of-the-Art Review,” The Scientific World Journal, Hindawi Publishing Corporation, Vol. 2014, Article ID. 538457, pp. 20, 2014.

(9) T Radford, S Damolini and J O'sullivan: “A Case Study on the Effect of Detailed 3D Finite Element Modelling on Nuclear Power Plant Building Response," 23rd Conference on Structural Mechanics in Reactor Technology, Vol.V, Manchester, United Kingdom, Paper ID. 738, 2015.
(10) AK Chopra : "Dynamics of Structures", Prentice Hall, 2014.

(11)H Roh, H Lee and JS Lee : "New Lumped-Mass-Stick Model based on Modal Characteristics of Structures: Development and Application to a Nuclear Containment Building,” Earthquake Engineering and Engineering Vibration, Vol. 12, No. 2, pp. 307-317, 2013.

(12)H Lee, YC Ou, H Roh and JS Lee : "Simplified Model and Seismic Response of Integrated Nuclear Containment System based on Frequency Adaptive Lumped-Mass Stick Modeling Approach,” KSCE Journal of Civil Engineering, Vol. 19, No. 6, pp. 1757-1766, 2015. 